\title{
Gradient de distribution géographique de la mutation principale de la mucoviscidose en Europe
}

\begin{abstract}
1. Boat TF, Welsh MJ, Beaudet AI. Cystic fibrosis. In : Scriver I), el al. The Melabolic Basis of Inherited Disease, $6^{\mathrm{e}}$ ed. New York : McGrawIHill, 1991: 2649-80.

2. Kerem B, Rommens JM, Buchanam JA, el al. Identification of the cystic fibrosis gene : genetic analysis. Science $1989 ; 245: 1073-80$.

3. Mc Intosh I, Lorenzo ML, Brock IJH. Frequency of $\Delta \mathrm{F} 508$ mutation on cystic fibrosis chromosomes in UK. Lancet 1989 ; i : 1404-5.

4. Estivill X, Chillon M, Casals ' $T$, et al. $\Delta \mathrm{F} 508$ gene deletion in cystic fibrosis in southern Europe. Lancel 1989 ; i, 1404

5. Romeo G, Devoto M. Population analysis of the major mutation in cystic fibrosis Hum Gene $1990 ; 85: 391-445$
\end{abstract}

6. The cystic fibrosis genetic consortium. Worldwide surveny of the $\Delta \mathrm{l} 5008$ mutation. $\mathrm{Am} \mathrm{J} \mathrm{Hum}$ Genet $1990 ; 47$ : 359-69.

7. The identification of the $\mathrm{CF}$ gene : recent progress and new research strategies. Workshop at Sestri I evante, 9-11 avril, 1990.

8. Lucotte G, Hazout S, Isirat F. Northwest/south-east gradient in $\Delta \mathrm{F} 508$ frequency in Europe. Lancet 1991; i : 882-3.

9. Lucotte G, Hazout S. A NW-SE decreasing gradient of the $\Delta \mathrm{F} 508$ frequencies in Europe. ECCFC Newslett 1990; 1: 2-3.

10. European working group of $\mathrm{Cl}$ genetics. Gradient of distribution in Europe of the major $\mathrm{CF}$ mutation and of its associated haplotype. Hum Genet 1990 ; 85 : 436-41.

11. Piazza A, Memozzi P, Cavalli-Sforza LL. The making and testing of geographic genefrequency maps. Biometrics 1991; 37 : 635-59.

12. Wijsman EM, Cavalli-Sforza LL. Migration and genetic population structure with special reference to humans. Ann Rev Ecol Syst 1984; 15 279-301.

13. Sokal RR, Harding RM, Oden NL. Spatial pattern of human gene frequencies in Europe. Am J Phys Anthropol 1989;80: 267-94.

14. Menozzi P, Piazza A, Cavalli-Sforza LI. Synthetic maps of human gene frequencies in Europeans. Science 1978; 201: 786-92.

15. Sokal RR, Oden NL, Wilson C. Genetics evidence for the spread of agriculture in Europe
La mucoviscidose est la plus fréquente en Europe des maladies héréditaires à déterminisme autosomique récessif [1], avec une incidence de l'ordre de $1 / 2500$ et une fréquence des porteurs dans la population voisine de 1/30. Le clonage du gène impliqué [2] a permis de montrer que la mutation la plus fréquente (la délétion $\Delta \mathrm{F} 508$ ) était présente à $68 \%$ dans la collection initiale des familles de mucoviscidose de Toronto, a priori très hétérogène cependant du point de vue ethnique.

Deux publications préliminaires ont concerné la fréquence de la $\Delta \mathrm{F} 508$ parmi les chromosomes mucoviscidosiques en Europe: pour environ 100 familles de mucoviscidose en Écosse la fréquence de la $\Delta \mathrm{F} 508$ est de $74,4 \%$ [3], alors qu'elle n'est que de 46,2\% pour 736 chromosomes dans des familles espagnoles et italiennes [4]. De nos jours, la fréquence de la mutation $\Delta$ F508 est bien connue pour différents pays d'Europe $[5,6,7]$; nous confirmons ici, et ce de façon détaillée, que la fréquence de la $\Delta \mathrm{F} 508$ parmi les chromosomes mucoviscidosiques varie selon les différentes populations européennes, étant plus élevée chez les Européens du nord que chez ceux localisés au sud de ce continent.

Le Tableau I résume les résultats des travaux à ce sujet obtenus récemment dans 44 laboratoires différents. Ces fréquences ont été sélectionnées sur la base de leurs représentativités, pour des données non-chevauchantes réparties dans différents pays ou régions d'Europe. Y sont aussi indiqués, à titre de comparaison, des résultats partiels concernant l'URSS, ainsi que ceux pour certaines communautés arabes vivant en Israël et en Algérie.

La figure 1 donne une représentation des lignes d'isofréquence de la $\Delta \mathrm{F} 508$, superposées sur la carte de l'Europe
[8], selon notre méthode originale de calcul [9]. Les données présentées montrent qu'il existe des variations considérables dans la fréquence de la $\Delta F 508$, avec aux deux extrêmes des valeurs minimales de 0,26 en Yougoslavie du sud et maximales $(0,88)$ au Danemark (les îles Féroé sont une dépendance du Danemark); des fréquences basses de la mutation sont observées dans les populations du SudEst, mais aussi pour une partie de l'Irlande (Nord-Ouest) ainsi qu'en Suède et en Finlande (Nord-Est). Cependant, la particularité la plus importante visible dans le dessin de répartition des fréquences est la démonstration claire d'un gradient décroissant de ces valeurs selon l'axe nord-ouest - sud-est pour la partie continentale de l'Europe ; cette singularité avait déjà été montrée précédemment [10] sur la base de données plus partielles que celles présentées ici, et par utilisation d'une autre méthode de construction de la carte [11] que celle que nous avons adoptée.

A l'évidence, cette variation clinale de fréquence décroissante selon le plus grand axe de l'Europe (si l'on inclut l'Islande et la Turquie et en constatant que les données relatives à l'URSS sont très peu représentées) indique un foyer nordique de la $\Delta \mathrm{F} 508$ et une diffusion par flux génique de la mutation vers les contrées plus sudistes. Il existe, en génétique des populations, un modèle dit "d'isolement par distance "qui peut rendre compte de cet aspect singulier observé, en particulier la modalité de la diffusion interdémique persistante sur de longues durées [12] à partir d'une population d'origine, sous l'influence d'un flux directionnel de gène sur tout ou partie d'un continent. Encore faudrait-il démontrer sur une base 
Tableau I

DISTRIBUTION GÉOGRAPHIQUE DE LA FRÉQUENCE DE LA MUTATION $\triangle$ F508 DANS DIFFÉRENTES POPULATIONS D'EUROPE (LES DONNÉES CONCERNENT 24 PAYS ÉCHANTILLONNÉS)

\begin{tabular}{|c|c|c|c|c|c|c|}
\hline & Pays & Villes ou régions & $\mathbf{N}$ & $\mathbf{n}$ & $f$ & Investigateur principal \\
\hline 1 & Finlande & Helsinki & 40 & 18 & 0,45 & de La Chapelle \\
\hline 2 & Suède & Stockholm & 154 & 81 & 0,52 & Anvret \\
\hline 3 & Danemark & $\begin{array}{l}\text { Copenhague } \\
\text { lles Féroé }\end{array}$ & $\begin{array}{r}423 \\
24\end{array}$ & $\begin{array}{r}373 \\
24\end{array}$ & $\begin{array}{l}0,88 \\
1\end{array}$ & $\begin{array}{l}\text { Schwartz } \\
\text { Sorensen }\end{array}$ \\
\hline 4 & Irlande & $\begin{array}{l}\text { Belfast } \\
\text { Dublin }\end{array}$ & $\begin{array}{l}204 \\
120\end{array}$ & $\begin{array}{r}111 \\
91\end{array}$ & $\begin{array}{l}0,54 \\
0,76\end{array}$ & $\begin{array}{l}\text { Graham } \\
\text { Humphries }\end{array}$ \\
\hline 5 & Angleterre & $\begin{array}{l}\text { Edimbourg } \\
\text { Manchester } \\
\text { Londres }\end{array}$ & $\begin{array}{r}238 \\
600 \\
87\end{array}$ & $\begin{array}{r}175 \\
479 \\
75\end{array}$ & $\begin{array}{l}0,74 \\
0,80 \\
0,86\end{array}$ & $\begin{array}{l}\text { Mclntosh } \\
\text { Super } \\
\text { Williamson }\end{array}$ \\
\hline 6 & Pologne & Varsovie & 22 & 12 & 0,55 & Maciejko \\
\hline 7 & Allemagne & $\begin{array}{l}\text { Est } \\
\text { Berlin } \\
\text { Bonn }\end{array}$ & $\begin{array}{l}314 \\
662 \\
204\end{array}$ & $\begin{array}{l}194 \\
492 \\
164\end{array}$ & $\begin{array}{l}0,62 \\
0,74 \\
0,80\end{array}$ & $\begin{array}{l}\text { Coutelle } \\
\text { Schmidtke } \\
\text { Olek }\end{array}$ \\
\hline 8 & Hollande & $\begin{array}{l}\text { Groningen } \\
\text { Rotterdam }\end{array}$ & $\begin{array}{l}166 \\
235\end{array}$ & $\begin{array}{l}125 \\
185\end{array}$ & $\begin{array}{l}0,75 \\
0,79\end{array}$ & $\begin{array}{l}\text { Scheffer } \\
\text { Oostra }\end{array}$ \\
\hline 9 & Belgique & $\begin{array}{l}\text { Bruxelles } \\
\text { Louvain } \\
\text { Liège }\end{array}$ & $\begin{array}{l}150 \\
124 \\
214\end{array}$ & $\begin{array}{r}116 \\
80 \\
166\end{array}$ & $\begin{array}{l}0,77 \\
0,65 \\
0,78\end{array}$ & $\begin{array}{l}\text { Lissens } \\
\text { Cassiman } \\
\text { Vassart }\end{array}$ \\
\hline 10 & Tchécoslovaquie & $\begin{array}{l}\text { Prague } \\
\text { Bratislava }\end{array}$ & $\begin{array}{r}354 \\
46\end{array}$ & $\begin{array}{r}240 \\
29\end{array}$ & $\begin{array}{l}0,68 \\
0,63\end{array}$ & $\begin{array}{l}\text { Macek } \\
\text { Ferak }\end{array}$ \\
\hline 11 & Autriche & Vienne & 120 & 94 & 0,78 & Spona \\
\hline 12 & Suisse & Zürich & 394 & 232 & 0,69 & Malik \\
\hline 13 & France & $\begin{array}{l}\text { Paris } \\
\text { Brest } \\
\text { Lyon } \\
\text { Grenoble } \\
\text { Montpellier }\end{array}$ & $\begin{array}{r}162 \\
224 \\
332 \\
90 \\
129\end{array}$ & $\begin{array}{r}91 \\
181 \\
261 \\
62 \\
81\end{array}$ & $\begin{array}{l}0,77 \\
0,81 \\
0,79 \\
0,69 \\
0,63\end{array}$ & $\begin{array}{l}\text { Lucotte } \\
\text { Ferec } \\
\text { Bozon } \\
\text { Lunardi } \\
\text { Claustres }\end{array}$ \\
\hline 14 & Portugal & Lisbonne & 84 & 45 & 0,54 & Duarte \\
\hline 15 & Espagne & $\begin{array}{l}\text { Barcelonne } \\
\text { Palma }\end{array}$ & $\begin{array}{r}466 \\
15\end{array}$ & $\begin{array}{r}239 \\
11\end{array}$ & $\begin{array}{l}0,51 \\
0,73\end{array}$ & $\begin{array}{l}\text { Estivill } \\
\text { Joame-Roïg }\end{array}$ \\
\hline 16 & Italie & $\begin{array}{l}\text { Milan } \\
\text { Turin } \\
\text { Vérone } \\
\text { Génes } \\
\text { Rome } \\
\text { Naples } \\
\text { Sardaigne }\end{array}$ & $\begin{array}{r}286 \\
40 \\
250 \\
122 \\
350 \\
102 \\
42\end{array}$ & $\begin{array}{r}156 \\
20 \\
90 \\
57 \\
157 \\
55 \\
24\end{array}$ & $\begin{array}{l}0,55 \\
0,50 \\
0,36 \\
0,47 \\
0,45 \\
0,53 \\
0,57\end{array}$ & $\begin{array}{l}\text { Ferrari } \\
\text { Carbonara } \\
\text { Pignati } \\
\text { Romeo } \\
\text { Novelli } \\
\text { Catiglione } \\
\text { Rostelli }\end{array}$ \\
\hline 17 & Yougoslavie & $\begin{array}{l}\text { Nord } \\
\text { Sud }\end{array}$ & $\begin{array}{l}39 \\
34\end{array}$ & $\begin{array}{r}15 \\
9\end{array}$ & $\begin{array}{l}0,38 \\
0,26\end{array}$ & $\begin{array}{l}\text { Efremov } \\
\text { Komel }\end{array}$ \\
\hline 18 & Bulgarie & Sofia & 110 & 64 & 0,58 & Kalaydjieva \\
\hline 19 & Albanie & Tirana & 39 & 15 & 0,38 & Simova \\
\hline 20 & Russie & Odessa & 58 & 26 & 0,44 & Romeo \\
\hline 21 & Grèce & Athènes & 194 & 105 & 0,54 & Balassopoulos \\
\hline 22 & Turquie & Istambul & 38 & 8 & 0,26 & Tummler \\
\hline 23 & Israël & Tel-Aviv & 23 & 5 & 0,21 & Lerer \\
\hline 24 & Algérie & Alger & 48 & 21 & 0,43 & Lucotte \\
\hline
\end{tabular}

$N$ : nombres de chromosomes mucoviscidosiques testés; $n$ : nombres de ceux d'entre eux portant la mutation $\Delta F 508$; $f$ : fréquences. $\mathrm{m} / \mathrm{s} n^{\circ} 1$, vol. 8, janvier 92 
quantitative tangible que la différenciation à longue distance obscrvéc cst réellement d'allure clinale (cc qui est difficile, étant donné que les principaux effectifs dont on dispose concernent les villes, et même des capitalcs, considérécs comme représentant des valeurs moyennes dans les pays correspondants). Unc telle modalité de cline nord-ouest - sud-cst a d'aillcurs été déjà démontrée pour un nombre rclativement important de variants génétiques en Europe [13]. L'interprétation généralc que l'on en donne habituellement (bien que, dans le cas de la $\Delta \mathrm{F} 508$, il soit inversé) est cn relation avec la progression des techniques de l'agriculture au néolithique, par expansion démique à partir du MoyenOrient $[14,15]$.

G.L.

\section{AVIS AUX AUTEURS DE TRAVAUX IMPORTANTS}

$\mathrm{m} / \mathrm{s}$ propose aux auteurs de travaux importants, publiés dans es revues d'audience internatiole et de premier niveau, de résenter leurs résultats sous orme de brève, de nouvelle, voire de mini-synthèse, au mieux publiés dans médecine/sciences parallèlement à l'article princeps.

\section{LA RÉDACTION}

Les manuscrits doivent être adressés à : médecine/sciences, 6, rue Blanche, 92120 Montrouge, France.

Tél. : (1) 47.35.85.52

Fax : 46.57 .10 .09



Figure 1. Carte de fréquences de la mutation $\triangle F 508$ en Europe. Les points indiquent les villes échantillonnées, et les lignes, les niveaux d'isofréquences; la régression des fréquences de $\Delta F 508$ (pondérées selon les effectifs) a été calculée en fonction des coordonnées géographiques normalisées lla carte est centrée, dans un système d'axes arbitraire, sur la ville de Bernel, sous la forme d'un polynôme du $4^{e}$ degré décrit dans [9] et qui est de la forme : $P(X Y)=0,654-$ $0,380 X+0,473 Y-0,379 X^{2}+0,140 X Y+0,151 Y^{2}+0,376 X^{3}-$ $0,605 X^{2} Y+0,456 X Y^{2}-0,432 Y^{3}+1,406 X^{4}+1,298 X^{3} Y-$ $2,694 X^{2} Y^{2}-2,408 X Y^{3}+0,751 Y^{4}$.

\section{ERRATUM}

Nous republions ici la figure 1 de la mini-synthèse : " les circuits de la mémorisation sous le scalpel des dissecteurs " $\left(\mathrm{m} / \mathrm{s} n^{\circ} 10, \mathrm{vol} .7, p\right.$. 1069), dans laquelle les flèches se trouvaient mal positionnées.

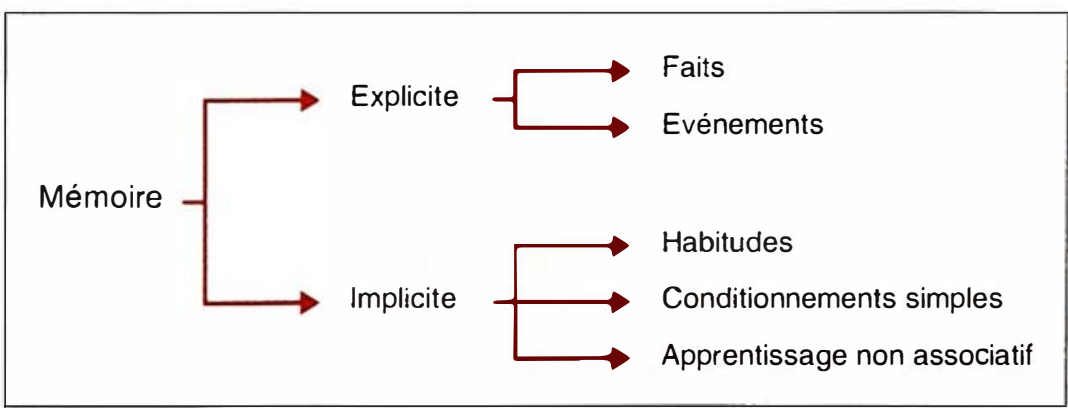

Figure 1. Schéma présentant la classification des diverses formes de " mémoire ". La mémoire explicite lou "déclarative ") qui concerne les faits et les événements que l'on peut rappeler consciemment est opposée à la mémoire implicite (ou " non-déclarative ") qui renvoie à l'ensemble des éléments mémorisés dans le cadre d'apprentissages comportementaux, éléments que l'on ne peut rappeler de façon consciente mais qui se révèlent dans l'utilisation des capacités acquises. 